\title{
Fast iterative reconstruction for helical pinhole SPECT imaging
}

\author{
Po-Chia Huang and Ching-Han Hsu * \\ Department of Biomedical Engineering and Environmental Sciences, National Tsing-Hua University, \\ Hsinchu, Taiwan
}

\begin{abstract}
Pinhole SPECT for small animal has become a routine procedure in many applications of molecular biology and pharmaceutical development. There is an increasing demand in the whole body imaging of lab animals. A simple and direct solution is to scan the object along a helical trajectory, similar to a helical CT scan. The corresponding acquisition time can be greatly reduced, while the over-lapping and gap between consecutive bed positions can be avoided. However, helical pinhole SPECT inevitably leads to the tremendous increase in computational complexity when the iterative reconstruction algorithms are applied. We suggest a novel voxel-driven (VD) system model which can be integrated with geometric symmetries from helical trajectory for fast iterative image reconstruction. Such a model construction can also achieve faster calculation and lower storage requirement of the system matrix. Due to the independence among various symmetries, it permits parallel coding to further boost computation efficiency of forward/backward projection. From phantom study, the results also indicate that the proposed VD model can adequately model the helical pinhole SPECT scanner with manageable storage size of system matrix and clinically acceptable computation loading of reconstruction.
\end{abstract}

Keywords: Pinhole SPECT, helical trajectory, voxel-driven system model, ray-tracing algorithms

\section{Introduction}

Pinhole SPECT has become a powerful tool for small animal studies [1-4]. Conventional usage of pinhole SPECT is to scan an object around a circular trajectory. Since the pinhole SPECT around a circular orbit can only scan a cubic-like volume, it can merely provide a very limited field-of-view (FOV). If one needs to scan a longer object, it may require to scan multiple bed positions to cover the whole object [5]. The advantage of multi-bed method is that the data acquired in each bed position can be reconstructed independently and then the resultant images can be stacked up into the final image volume. However, the longer acquisition time is its shortage.

There are still some accuracy issues concerning the multi-bed method. If the distance between two adjacent bed positions is too close, there will be some overlapped regions, as shown in Figure 1 (a), and increase scan time. On the contrary, if the distance is too long, the acquired data may lose a portion of projection data, as shown in Figure 1 (b). Such a missing data could make the acquired multi-bed data no

\footnotetext{
*Address for correspondence: Ching-Han Hsu, Department of Biomedical Engineering and Environmental Sciences, National Tsing-Hua University, Hsinchu, Taiwan. Tel.:886-3-5752416; Fax: 886-3-5718649; E-mail: cghsu@mx.nthu.edu.tw.
} 


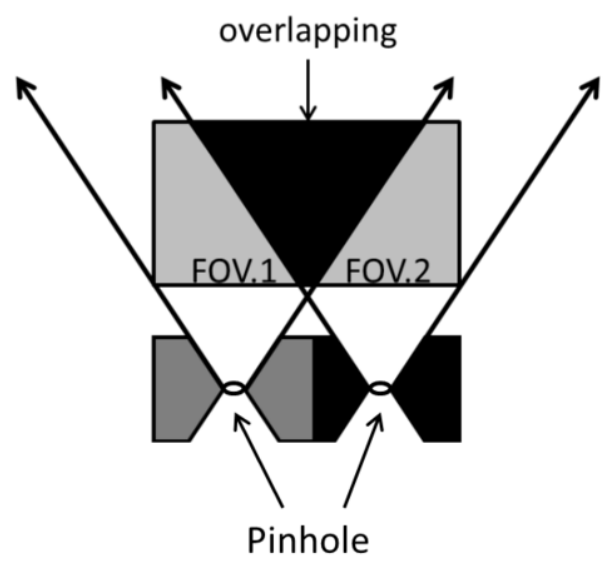

(a)

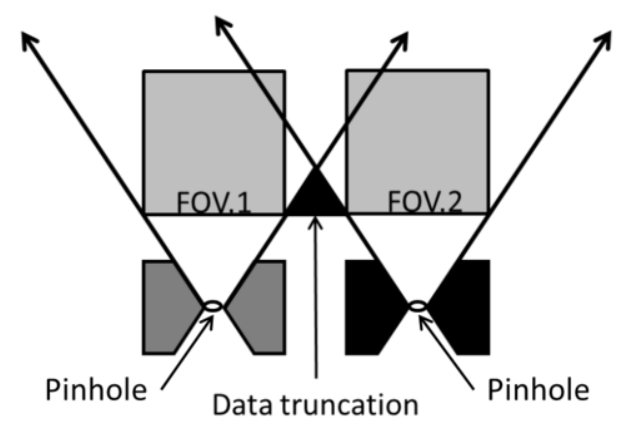

(b)

Fig. 1. Scanning a long object using a conventional pinhole SPECT at multiple bed positions. (a) When two adjacent bed positions are too close, there will exist some overlapping which decreases the efficiency of detector usage. (b) When two adjacent bed positions are too distal, there will exist the issue of data truncation.

longer comply with the the Kirllov-Tuy condition [6] and would lead to incomplete data problem during image reconstruction stage. Therefore, for imaging longer objects, helical pinhole SPECT becomes a more appropriate alternative than conventional multi-bed method.

A helical SPECT can not only increase the FOV, but also provides many other advantages [5,7]. First, the image truncations of multi-bed pinhole SPECT will not occur in helical pinhole SPECT. Second, the helical pinhole SPECT can also avoid the axial blurring which is mainly due to the incomplete sampling in a multi-bed scan. Finally, the helical pinhole SPECT can reduce the effect that photon emission rate decay during the scan of a longer object.

In this work, we plan to investigate whether iterative reconstructions are feasible for helical SPECT. For statistical/iterative reconstruction algorithms, the system matrix, which records the physical processes of single photons from their emission and detection, plays an important role [8]. There are many methods to estimate this system matrix. A more accurate approach to construct such a system matrix is to measure the point spread function either by using real point source or through Monte-Carlo simulation $[9,10]$. This approach can generate a accurate matrix and it also contains all intrinsic characteristics of scanner, such as detector efficiency. But, both of the measurement or simulated methods are very time consuming. In reality, it is almost impossible to measure or simulate all the points in the FOV. Many interpolation algorithms are proposed to calculate the missing terms on those locations where the system response functions are not measured $[2,11]$.

The second category to estimate the system matrix is based on geometric calculation [12-14]. The ray-tracing algorithm (RT) is a another popular method to estimate the system matrix $[15,16]$. The RT algorithm use the intersection of a ray passing through one voxel as the photon detection probability. It is an efficient method because this algorithm use only by the four basic arithmetic operations. Nevertheless, the RT algorithm can not completely model the non-parallel projection system, such as pinhole SPECT. In addition, the use the single-ray ray-tracing (SRRT) to model the pinhole finite aperture as an ideal pinhole is less appropriate.

A more suitable method to describe the finite aperture is multi-ray ray-tracing (MRRT) method $[1,17]$. One major limitation of the MRRT is the loss of voxel during projection operations. Consider two distinct 


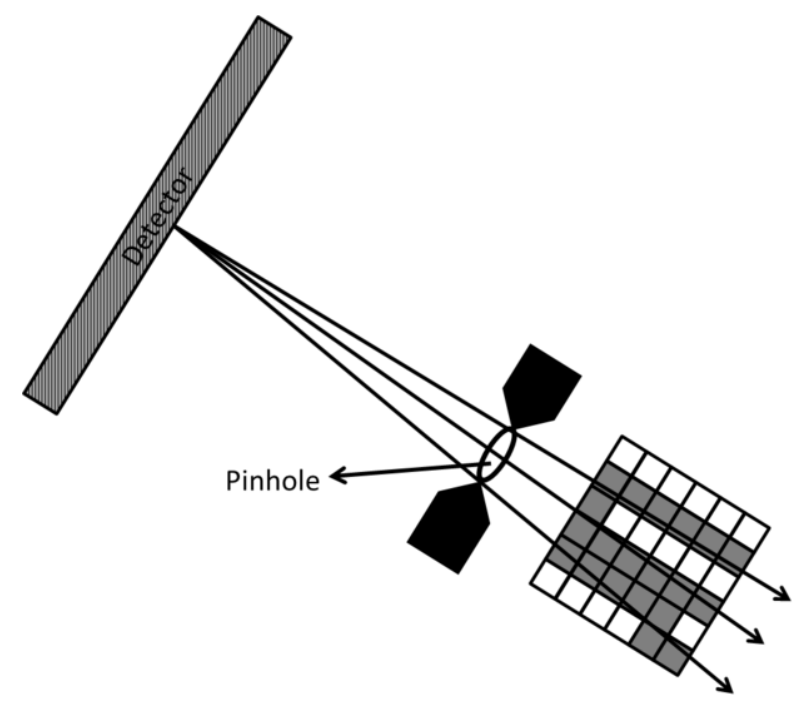

Fig. 2. The schematic diagram for the losing projection information by using RT method. The voxel with black should be detected but RT method can not trace them.

rays through the aperture center, as shown in Figure 2. The distance between two distinct rays increases as the rays travel away from the aperture. This distance increasing may lead to the loss of projection information of some voxels which are far away to the origin of the ray Nevertheless, the MRRT also can not model precisely the finite aperture, because it may requires many rays fill the entire pinhole aperture.

Finally, these RT algorithms are not depth sensitive. In pinhole SPECT system, the system sensitivity depend on the incident angles defined by the pinhole collimator and the voxel locations. When a voxel is located closer to the collimator, the photons emitted from the the voxel can be more easily detected (i.e., more sensitive) than those voxels far away from the collimator. The RT algorithms assume uniform sensitivity for all voxles along a ray and fail to model the depth-independent characteristics of SPECT systems.

Unlike the large time consumption of the measurement methods and the depth-independence of the RT methods, a voxel-driven (VD) model could adequately describe the characteristics of photon detection in a pinhole SPECT system [18]. The VD model is based on geometric calculation, so that the VD model can easily model the finite-aperture and depth-dependent effects in pinhole SPECT. The VD model is to use the cone section to calculate the projection, both normal and tilted pinhole systems are directly applicable. Since the VD model is based on geometric calculation, the system matrix can be constructed according to the resolution specification. The computational loading for the VD model is slightly greater than that of the RT algorithms, but the accuracy of the VD model is better than RT model. In addition, the VD model can easily incorporate other physical factors, like the penetration effect in pinhole edge once the photon energy and the attenuation coefficient of collimator metal are known.

The most challenge task in system matrix is its storage size and computational loading. For the iterative image reconstruction of a helical SPECT scan, the required system matrix performs the mapping forward and backward between the entire image volume and the whole projection data simultaneously. It is not surprising that the storage of the system matrix is tremendous and the computation of projection operations is heavy. To cope with these challenges, we will exploit the geometric symmetries to reduce the storage size and implement the multi-threaded program to accelerate the projection operations. 


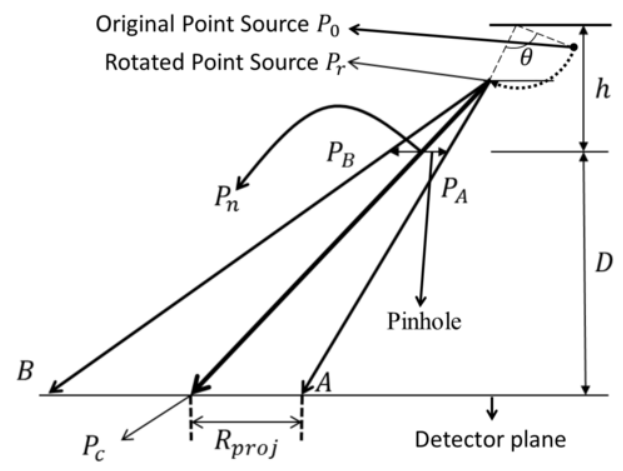

Fig. 3. When detector and pinhole plane are parallel, the projection shape is similar to the pinhole. Suppose a point source is located on $P_{0}\left(x_{0}, y_{0}, z_{0}\right) . P_{r}$ is the position for the point source after rotating with angle $\theta . P_{n}$ and $P_{c}$ denote the coordinates of pinhole center and projection center, respectively.

The paper is organized as follows. We start with the derivation of the proposed VD model and its geometric characteristics in Section 2. Section 3 presents numerical experiments and performance evaluation of the proposed system model. Detailed discussions about memory storage and implementation are given in Section 4. Finally, the conclusions are drawn in Section 5.

\section{Materials and methods}

\subsection{The proposed VD model}

For iterative reconstruction for a pinhole SPECT scanner, we propose to construct a VD model based on the configuration of scanner's geometry. Suppose that each image voxel denotes a point source. The projection of a point source passing through the pinhole collimator can be well modelled by a cone section. There exist three possible cone shapes. The first type is the case when the pinhole plan and detection plane are parallel to each other. The projection shape is approximate to to the pinhole shape. For a circular pinhole, a schematics diagram is shown in Figure 3. For the other two types, they only occur when detector and pinhole planes are not parallel. The resultant shape is either an ellipse or a dualhalf-ellipses [19], In this work, only single pinhole collimator is considered. We evaluate the feasibility of iterative image reconstruction, particularly on issues like storage size and computational load related to projection matrix.

For the single pinhole collimator, when the detection plane is parallel to the pinhole plane, the projection shape of each voxel becomes a circle [18]. Consider a voxel $V_{0}$ with coordinates $\left(x_{0}, y_{0}, z_{0}\right)$. Let $\left(x_{p}, y_{p}, z_{p}\right)$ denote the center of the pinhole aperture. The projection of the voxel $V_{0}$ has the center located at:

$$
\begin{aligned}
& x_{\text {proj }}=\frac{\left(h+d-z_{p}\right) \times\left(x_{0} \cos \theta+z_{0} \sin \theta-x_{p}\right)}{x_{0} \sin \theta-z_{0} \cos \theta-z_{p}}+x_{p} \\
& y_{\text {proj }}=\frac{\left(h+d-z_{p}\right) \times\left(y_{0}-y_{p}\right)}{x_{0} \sin \theta-z_{0} \cos \theta-z_{p}}+y_{p} \\
& z_{\text {proj }}=h+d
\end{aligned}
$$




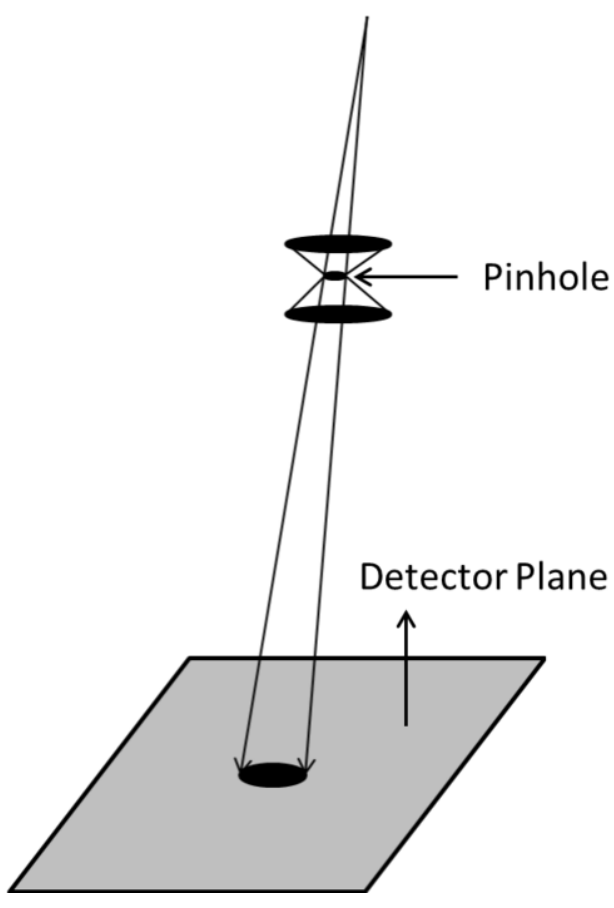

(a)

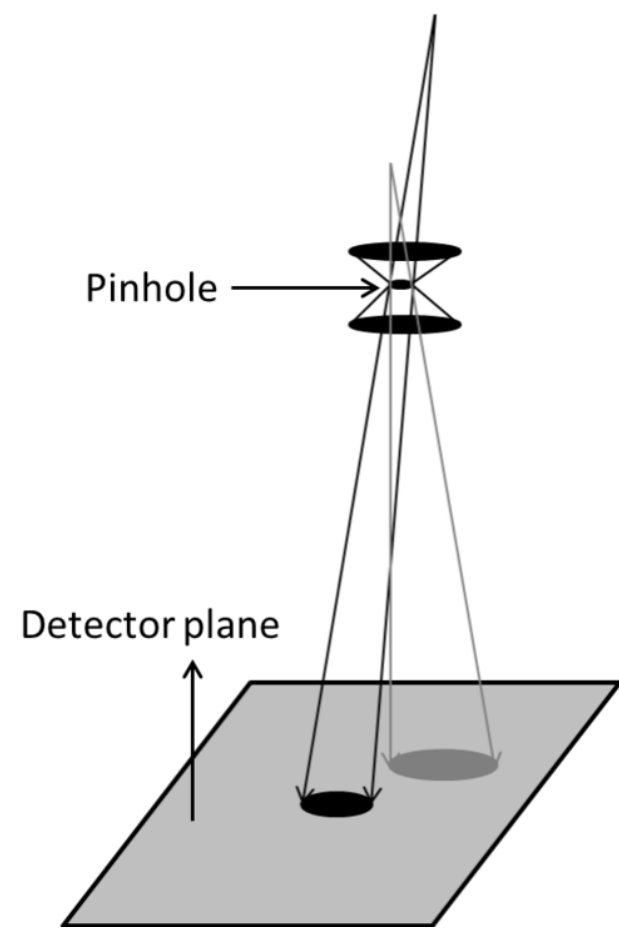

(b)

Fig. 4. The voxel-driven model can include (a) the finite aperture and (b) the depth effect in pinhole SPECT system.

where $h$ is the radius of the rotation and $d$ the focal length. The camera is rotating along the $y$-axis in clockwise direction with $\theta$ representing the projection angle.

The corresponding radius of the projection circle can be derived as the following. In pinhole imaging, the magnification $M$ of each voxel depends on its depth and is defined by:

$$
M=\frac{d+h-x_{0} \sin \theta+z_{0} \cos \theta}{h-x_{0} \sin \theta+z_{0} \cos \theta}
$$

If the radius of the pinhole aperture is $R_{p}$, the corresponding radius of the projection circle is

$$
R_{\text {proj }}=R_{p} \times M
$$

We estimate the detection probability value from each voxel onto the detection plane based on the computed projection areas. Please refer to [18] for the detailed calculation. Since this detection probability is based on the geometric calculation, various geometrical and physical properties can be directly included to this model, i.e., the finite aperture pinhole and depth-dependent effects $[18,19]$, as shown in Figure 4.

\subsection{Geometric symmetries and multi-threaded computation}

Although a helical trajectory pinhole SPECT can cover larger image volume, the increased volume leads to the increased voxel number. Since the system storage size and computational loading are also di- 

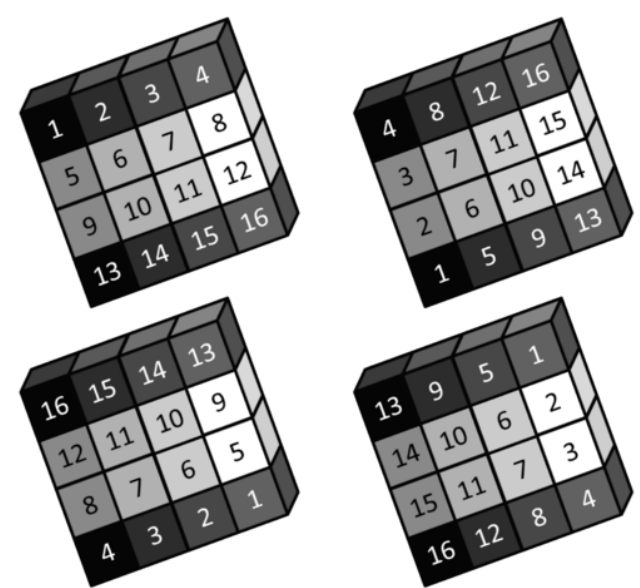

Fig. 5. A set of voxels on different projection angles with distinct voxel indices but sharing the same projection value. This symmetry mechanism is rotational symmetry.

rectly proportional to voxel numbers, they become the most challenging obstacle in image reconstruction. For an high efficient computation and storage, these symmetries can be used to cope with this challenge.

There are two type geometrical symmetries in the helical trajectory pinhole SPECT. The first symmetry is the rotational symmetry (RS) as conventional circular trajectory pinhole SPECT [19,20]. Since the pinhole SPECT has a cubic field of view (FOV), after rotating 90 degrees, the detection probabilities will be exactly the same as those probabilities of the 0 degree except for different voxel indecies. As shown in Figure 5, for some different voxels from certain specific projection angles, they will also share the same detection probabilities. It only requires to record the 90 degrees projection values, i.e., a 4-fold symmetry $[18,19]$. Such a rotational symmetry exists in all cubic voxel.

The second type of symmetry is the translational symmetry (TS). This symmetry only exists in helical scan mechanism. When the number of image volume is an integer multiple of the number of helical projection, the corresponding TS will exist, as shown in Figure 6. For example, the detection probabilities starting from 720 to 810 degrees can be considered as a shifted duplicate of the detection probabilities from 0 to 90 degrees. For the configuration of helical pinhole SPECT used in this research, the rotation angles are $4 \times 360=1440$ degrees in total. This means that there exists 4 -fold TS along the axial direction.

Since the RS is based on rotation scanner but TS is based on the object translation, these two symmetries are independent to each other. Therefore, there are total $4 \times 4=16$-fold symmetry in this system. During the operations of forward and backward projections in image reconstruction, they could be partitioned directly into sixteen mutually exclusive threads under a multiple core workstation to enhance reconstruction speed. The images results are reconstructed by a workstation with single Intel i7-2600 (3.4GHz) CPU and 16 GB RAM.

\section{Results}

In this work, we simulate the hardware parameters of a preclinical Nano SPECT/CT (Bioscan, Inc.) . The helical data acquisition is defined as follows. The focal length is $126.5 \mathrm{~mm}$, while the radius of rotation is $45.0 \mathrm{~mm}$. There are $256 \times 256$ detectors on detector plane and the detector size is $1.0 \mathrm{~mm} \times$ $1.0 \mathrm{~mm}$. Single pinhole collimator imaging system is considered in this feasibility research. The pinhole is circular and has the diameter $1.5 \mathrm{~mm}$. There are 160 equally-spaced projections around 1440 degrees. 


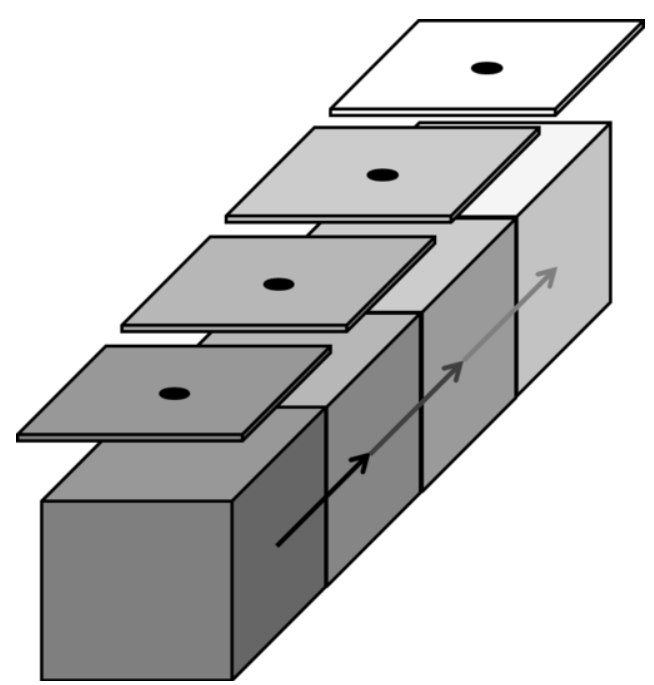

Fig. 6 . The translational symmetry: after rotating every 360 degrees, the relative position between object and pinhole is only a translational displacement.

We injected the radio-labeled (Tc-99m) tracer into two capillaries as two line sources. We placed one line source at the center of the rotation axis, and the other one $7.5 \mathrm{~mm}$ away from the center. The image results were reconstructed by applying the MLEM algorithm with 40 iterations [8]. The volume sizes are $128 \times 128 \times 320$ in total and the voxel width $0.4 \mathrm{~mm}$. The iterations are accelerated by a 16-thread code which corresponds to the total 16 -fold symmetry. Since 320 is a multiple to the projection number 160 , the translational symmetry exists in this study.

The acquisition projection data is shown in Figure 7. And the reconstructed images are shown in Figure 8. Since the pinhole imaging generates an inverse projection, the line source moves downward while the projection moves upward. In the reconstructed images, two line sources are maintaining parallel to each other and the distance between two lines in each image slice keeps the same. The feasibility result shows that the proposed VD model can correctly and effectively reconstruct image volume from the helical pinhole SPECT system.

\section{Discussions}

Without geometric symmetries, it requires 200 TB to store the original system matrix, which is impossible for memory storage or numerical computation. Due to the spareness nature of system matrix, it merely has to record only those non-zero probability. The corresponding storage size can be compressed down to $30 \mathrm{~GB}$ right away. Although the $30 \mathrm{~GB}$ seems to be manageable for a modern storage system, the direct matrix computation of forward and backward projections is still too time-consuming and less practical. If we further applied both RS and TS in storage, the storage size could shrink down to 1.88 GB. The final storage size is $1.88 \mathrm{~GB}$ which is a feasible size and suitable for routine reconstruction.

In addition, it only needs to record the detection probabilities for the angles starting from 360 to 450 degrees. For the rest of voxles at other projection angles, they can be directly and easily computed by index transformation based on the regularity of geometric symmetries. Consider an image volume which contains $m$ slices and each of slice has $n \times n$ voxels. Suppose that $\left(n_{x}, n_{y}, n_{z}\right)$ denotes the indices of a 


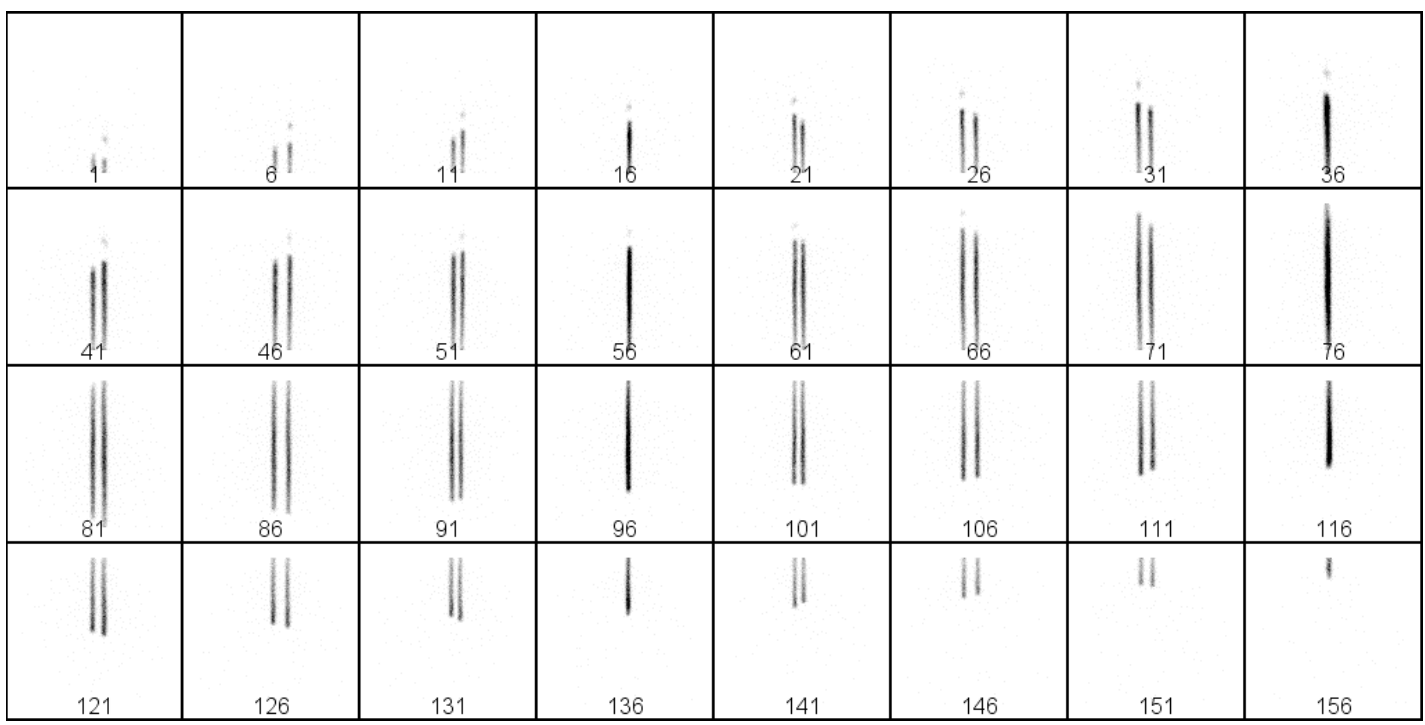

Fig. 7. Helical scans of two line sources. The corresponding projection angles are listed at the bottom of each box. As expected, there are 8 over-lapping between two lines.

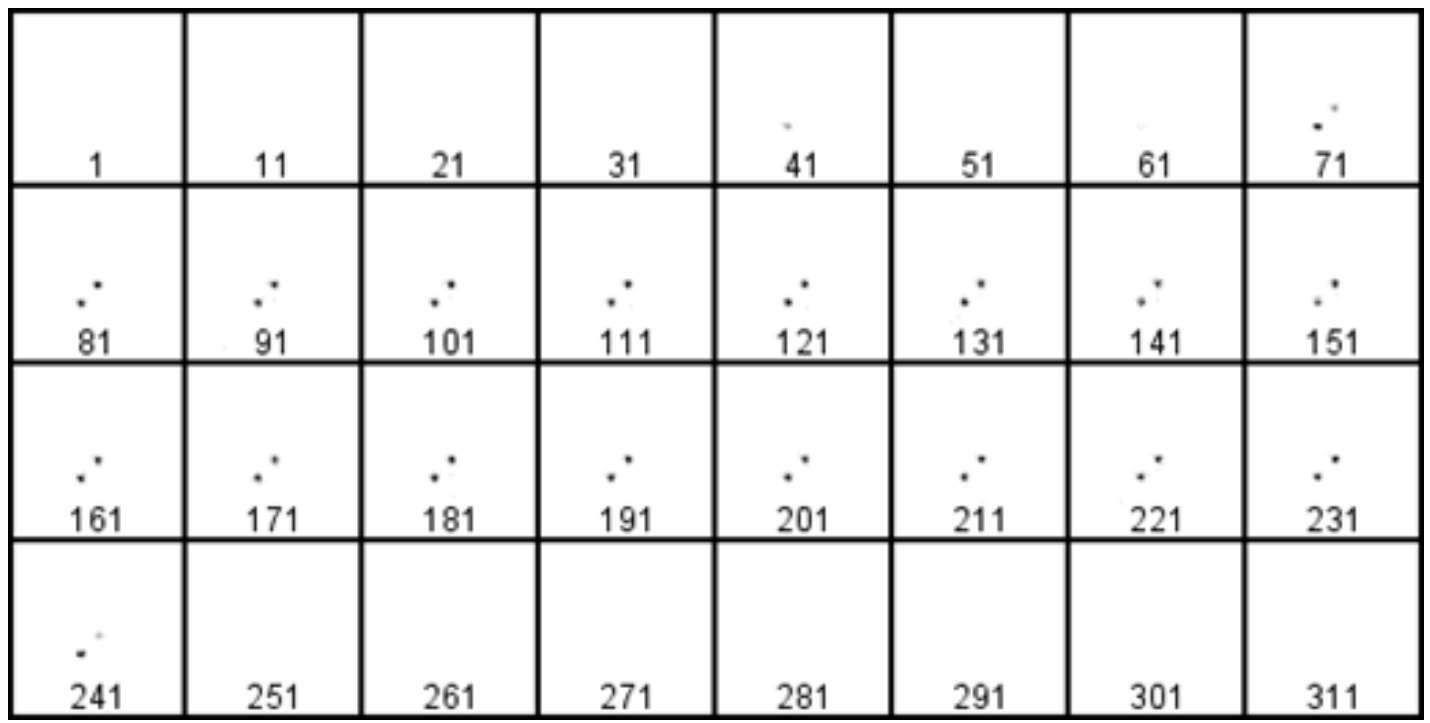

Fig. 8. The resultant images of two line sources after the MLEM image reconstruction with 40 iterations.

voxel $V$. If we expand the voxel into a one-dimensional array, then the indices of the voxel $V$ becomes

$$
N=n_{z} \times n \times n+n_{x} \times n+n_{y} .
$$

Given an voxel index $N_{\theta}$ after the detector plate rotates $\theta$ degree, then its 90 -degree symmetric point $N_{\theta+90}$ has the index given by: 


$$
\begin{aligned}
N_{\theta+90} & =\left[\left(N_{\theta}-1\right) / m\right] \times(n \times n) \\
& +\left[n-\left(N_{\theta} \% m\right) \% n-1\right] \times n+\left(N_{\theta} \% m\right) / n+1,
\end{aligned}
$$

where $\%$ denotes the arithmetic module operation. If we substitute $N_{\theta}$ by $N_{\theta+90}$ and $N_{\theta+180}$ and repeat the Eq. (7) twice, we can quickly compute the symmetric voxel indices $N_{\theta+180}$ and $N_{\theta+270}$ in projection angles $\theta+180$ and $\theta+270$ degrees, respectively.

The TS exists when the projection number per 360 degrees is an integer multiple of the slice number of the image volume. In this work, there are 320 slices in the image volume, while the projection number is $160 / 4=40$ per 360 degrees. Since $320=40 \times 8$, there exists the translational symmetry.

According to the reconstructed image, the vertical distance of two line sources is $5.4 \mathrm{~mm}$ and the horizontal distance is $5.2 \mathrm{~mm}$. The distance between two line sources is $7.50 \mathrm{~mm}$ as the phantom design. Since the line source is to inject the radio-labeled medicine into the capillaries, the spatial resolution is the same as the inner diameter of the capillaries. Therefore, the spatial resolution is about $1.0 \mathrm{~mm}$ as the capillaries inner diameter. The contrast recovery is the same for two line sources, in other words, these two line source have the same contract recovery in each slice.

By using the RS and TS, the size of system matrix becomes manageable. Since RS is generated on a 2D plane and TS is generated along the normal line of rotation plane, the RS and TS are independent. Hence, the projection operations can carried out in any multiple core work-station for fast computation. It takes about 12 minutes to reconstruct image using 40 MLEM iterations, which is acceptable for routine animal studies.

For multi-pinhole SPECT scanners with better system sensitivity, the proposed VD model can be directly applicable. The increase of storage size and computational time is approximately equal to the number of pinholes, since the projections of multiple pinholes are independent to each other.

\section{Conclusions}

In this paper, the feasibility of an iterative helical pinhole SPECT image reconstruction for small animal imaging has been investigated. We proposed a VD model to describe the detection probabilities of a helical pinhole SPECT scan. Since there are many geometric symmetries in helical SPECT system, the investigation suggest that it only needs to store projection information across 90 degrees. Furthermore, the concurrent nature of pinhole projections is ready to be fully exploited to accelerate the numerical computation by using multi-threaded codes. Other fast iterative algorithms, such as OSEM, are also suitable to applied the multi-threaded techniques. The experimental results also demonstrate that the proposed VD model can handle a longer object with manageable storage space and is clinically acceptable computation time. The proposed VD model can not only be extended to multiple pinhole collimator to increase system sensitivity, but also be integrated with other fast reconstruction algorithms.

\section{References}

[1] J. Nuyts, K. Vunckx, M. Defrise and C. Vanhove, Small animal imaging with multi-pinhole SPECT, Methods 48 (2009), $83-91$.

[2] M. A. Kupinski and H. H. Barrett, Small-animal SPECT imaging, Springer, 233 Spring Street, New York, NY 10013, USA, 2005 
[3] M.W. Lee and W.T. Lin and Y.C. Chen, Design optimization of multi-pinhole micro-SPECT configurations by signal detection tasks and system performance evaluations for mouse cardiac imaging, Physics in Medicine and Biology $\mathbf{6 0}(2015)$, $473-499$.

[4] K. Van Audenhaege, C. Vanhove, S. Vandenberghe and R. Van Holen, The evaluation of data completeness and image quality in multiplexing multi-pinhole SPECT, IEEE Transaction on Medical Imaging 34 (2014), $474-486$.

[5] S. D. Metzler, S. Vemulapalli, R. J. Jaszczak, G. Akabani and B. B. Chin, Feasibility of whole-body functional mouse imaging using helical pinhole SPECT, Molecular Imaging and Biology 12 (2010), $35-41$.

[6] H. K. Tuy, An inversion formula for cone-beam reconstruction, Applied Mathematics $\mathbf{4 3}$ (1983), $546-552$.

[7] S. Metzler, K. Greer and R. Jaszczak, Helical pinhole SPECT for small-animal imaging: a method for addressing sampling completeness, IEEE Transactions on Nuclear Science 50 (2003), $1575-1583$.

[8] C.H. Hsu, An Investigation of Block-Sequential Algorithms in Statistical PET Image Reconstruction, Journal of Medical and Biological Engineering 24 (2004), $69-75$.

[9] D. Lefkoupoulos, J. Fonroget, J. Y. Devaux, J. B. Guilhem, J. C. Rou-cayrol and R. Guiranud, Quantitative 3D imaging with coded apertures by using SVD decomposition of the transmission matrix, Proceedings of the Third World Congress of Nuclear Medicine and Biology, Paris, France, (1982), 503 - 506.

[10] L. R. Furenlid, D. W. Wilson, Y.C. Chen, H. Kim, P. J. Pietraski, M. J. Crawford and H. H. Barrett, FastSPECT II: a second-generation high-resolution dynamic SPECT image, IEEE Transactions on Nuclear Science 51 (2004), 631 - 635.

[11] R. K. Rowe, J. N. Aarsvold, H. H. Barrett, J.C. Chen, B. Moore, I. W. Pang, D. D. Patton and T. A. White, A stationary hemispherical SPECT Imager for Three-Dimensional Brain Imaging, The Journal of Nuclear Medicine 34 (1993), $474-$ 480.

[12] Z. E. Bitar, R. H. Huesman, R. Boutchko, V. Bekaert, D. Brasse and G. T. Gullberg, A detector response function design in pinhole SPECT including geometrical calibration, Physics in Medicine and Biology 58 (2013), 2395 - 2411.

[13] T. Dai, T. Ma, Q. Wei, H. Liu, X. Li, S. Wang and Y. Liu, Accurate image reconstruction based on standard-Gaussianmodel fitted system matrix in multipinhole small animal SPECT imaging, IEEE Nuclear Science Symposium and Medical Imaging Conference 1 (2013), $1-4$.

[14] B. Feng and G. Zeng, Modeling of pixelated detector in SPECT Pinhole Reconstruction, IEEE Transactions on Nuclear Science 61 (2014), $888-893$.

[15] R. L. Siddon, Fast calculation of the exact radiological path for a three-dimensional CT array, Medical Physics 12 (1985), $252-255$.

[16] G. Han, Z. Liang and J. You, A Fast Ray-Tracing Technique for TCT and RCT stidies, IEEE Nuclear Science Symposium and Medical Imaging Conference 3 (1999), 1515 - 1518.

[17] C. Vanhove, A. Andryev, D. M., J. Nuyts and A. Bossuyt, Resolution recovery in pinhole SPECT based on multi-ray projections: a phantom study, European Journal of Nuclear Medicine and Molecular Imaging 34 (2007), $170-180$.

[18] C. H. Hsu and P. C. Huang, A geometric system model of finite aperture in small animal pinhole SPECT imaging, Computerized Medical Imaging and Graphics 30 (2006), 181 - 185.

[19] P. C. Huang, C. H. Hsu, I. T. Hsiao and K. M. Lin, An accurate and efficient system model of iterative image reconstruction in high-resolution pinhole SPECT for small animal research, Journal of Instrumentation 4 (2009), P06007.

[20] V. Israel-Jost, P. Choquet, S. Salmon, C. Blondet, E. Sonnendrcker and A. Constantinesco, Pinhole SPECT imaging: compact projection/backprojection operator for efficient algebraic reconstruction, IEEE Transactions on Medical Imaging 25 (2006), 158 - 167. 